\title{
A Computer-Aided Ergonomic Assessment and Product Design System Using Digital Hands
}

\author{
Yui Endo ${ }^{1}$, Satoshi Kanai ${ }^{1}$, Takeshi Kishinami ${ }^{1}$, \\ Natsuki Miyata ${ }^{2}$, Makiko Kouchi ${ }^{2}$, and Masaaki Mochimaru ${ }^{2}$ \\ ${ }^{1}$ Hokkaido University, Japan \\ yendo@minf.coin.eng.hokudai.ac.jp \\ ${ }^{2}$ Digital Human Research Center, National Institute of \\ Advanced Industrial Science and Technology (AIST), Japan
}

\begin{abstract}
The purpose of this research is to develop a system for computeraided ergonomic assessment of products without real subjects and physical mockups by integrating a digital hand model with a product model. In previous work, we developed functions of a semi-automatic grasp planning for the digital hand and of quantitatively evaluating the grasp stability of the product based on the force-closure and the grasp quality in our system. We also confirmed the validity of the results of these functions by comparing them with the real grasp postures. In this paper, we propose a new function of evaluating "ease of grasping (EOG)" for the grasp posture based on EOG-map constructed from principal component analysis for finger joint angles in real subjects' grasps.
\end{abstract}

Keywords: Digital Human, Digital Hand, Computer Mannequin, Ease of Grasp, Principal Component Analysis.

\section{Introduction}

Recently, handheld information appliances such as mobile phones, handheld terminals and digital cameras etc. have widely spread to general users. Especially some of these appliances for professional use have to be operated with one grasp posture for long hours, so manufactures of these appliances have to take into consideration the ergonomic design. However, the ergonomic assessments of these appliances need the physical mockups and subjects for testing, and therefore take large time and cost. So there are an increasing need to do the ergonomic assessments in virtual environment using digital mockups of the appliances and digital human models especially including digital hand models.

Some simulation software using digital human models have been commercialized and are being used in the design of automobiles and airplanes [1]. However, the digital hand models included in the digital human models of such software do not necessarily satisfy desired accuracy and size variation of human hands when operating the handheld appliances. Moreover, the software does not have the functions of automatically generating the grasp postures, of evaluating the ease of grasp and of evaluating the ease of manipulations of the user-interface by fingers. 
Therefore, our research purpose is to develop a computer-aided ergonomic assessment and product redesign system for handheld information appliances using the digital hand model and the 3-dimesional product model of the appliance.

In our system, we realize the following feature functions for ergonomic assessment to satisfy our purpose:

1. Generation of kinematically and geometrically accurate 3-dimensional digital hand models with rich dimensional variation: We apply a digital hand model called "Dhaiba-Hand" [2] to the ergonomic assessment in our system.

2. Automatic generation and evaluation of the grasp posture: By only inputting a few user-interactions, our system automatically generates one of the possible grasp postures determined by the product shape and the digital hand geometry. It also quantitatively evaluates two indices of the grasp: i) grasp stability for the product geometry and ii) ease of grasping from aspect of finger joint angle configuration.

3. Automatic evaluation of easiness of the finger motions in operating the user interface: The system automatically moves fingers of the digital hand by following an operation task model of the user-interface (e.g. which button has to be pushed). It also automatically evaluates easiness of finger motions during operation of the user interface based on the flexion joint angles of fingers.

In this paper, we mainly discuss technical background of above second function especially of evaluating the "ease of grasping". This computer-aided ergonomic assessment system is being developed as a part of our government-funded project called "Sapporo IT Carrozzeria" [3] whose mission is developing rapid prototyping technology of information appliances.

\section{Previous Works}

\subsection{Related Works}

Some researches (for example, [4,5,6,7]) have proposed the generation and evaluation methods of the grasp postures of the digital hand for the objects. The methods of generating the grasp posture are roughly classified into two types: a variant method [5] and a generative method [4,6,7].

In the variant method, real grasp postures of many subjects for sample objects have been measured in advance using dataglove to build a grasp posture database. Then, in generation step, one grasp posture where his/her grasping object shape is most similar to the given object model shape is chosen from the grasp posture database. The selected posture is then modified to fit to the product model shape. If a very similar product shape can be found in the database, a nearly appropriate grasp posture for the given product can be obtained after this modification process.

On the other hand, in the generative method, the grasp posture is generated by full/semi-automatic grasping algorithm. This method does not need any database of the real grasp postures. For any unknown shape to be grasped, this method can generate a grasp posture which satisfying geometric boundary conditions when the hand is contacting with the product surface. 
However, the both of these researches did not discuss whether the obtained grasp postures are truly appropriate and possible one or not.

\subsection{Digital Hand Model and Grasp Stability Evaluation [6]}

\subsubsection{Digital Hand Model}

To perform effective digital ergonomic assessment, it is insufficient to use only one digital hand model with a fixed dimension because the physical dimensions of appliance users differ from person to person. Therefore, we needed to generate a digital hand model with possible anthropometric variation. In this purpose, we used a digital hand model based on the Dhaiba-Hand [2]. The digital hand model used in our system consists of the following four parts and relation among these parts is shown in Figure 1(b):

1. Link structure model: A link structure model approximates to the rotational motion of bones in the hand. The model was constructed from the measurement by MRI and the motion capture [8,9].

2. Surface skin model: A surface skin model is a 3-dimensional polygonal mesh for the hand surface generated from CT images, as shown in Figure 1(a). The geometry of the skin model is defined at only one opened posture.

3. Surface skin deformation algorithm: This algorithm defines the deformed geometry of the surface skin model when the posture of the link structure model is changed, as shown in Figure 1(b).

4. Finger closing motion sequencer: The finger closing motion sequencer is a function to automatically and naturally generate a finger-closing motion path of the hand model from a fully opened state to a clenched one, as shown in Figure 1(b). This motion reflects the joint angle constraints of the link structure model.

A link structure and a surface skin model are generated by inputting the 82 dimensional parameters of a specified subject's hand into the generic hand model
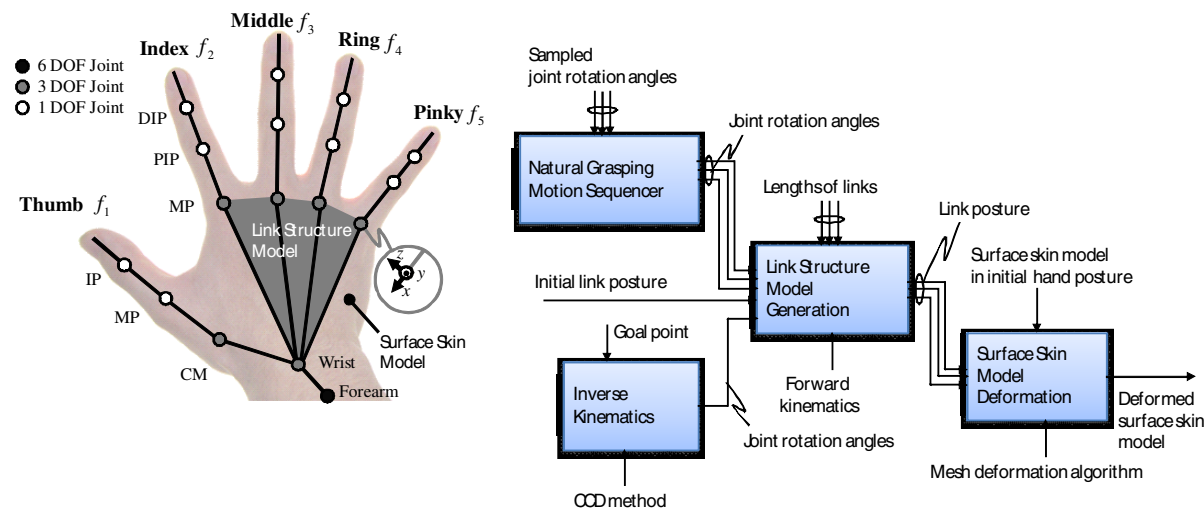

Fig. 1. The digital hand model of our system, (a) The link structure model, (b) The flow of the deformation of the surface skin model 
which are implemented in the Dhaiba-Hand [2]. On the other hand, a surface skin deformation algorithm and a finger closing motion sequencer were originally developed by us [6].

\subsubsection{Grasp Posture Generation and Evaluation of the Grasp Stability [6]}

The generation of the grasp posture of the digital hand model for the product shape model is the first step in our system. The process consists of four phases: 1) selection of the contact point candidates, 2) generation of the rough grasp posture, 3) optional correction of the contact points and 4) maximization of the number of the contact point.

After generating the grasp posture, the system automatically evaluates the grasp stability for the product in this estimated posture. We introduced the force-closure and the grasp quality into the evaluation of the grasp stability [10,11].

We also described the verification results of our system by comparing the estimated grasp postures given from the system with the ones from experiments, and also by comparing the grasp stability evaluated by our system with the ones felt by real subjects.

\subsubsection{The Problems on Generating Grasp Postures and Our Solution}

As described in the previous section, our generation and evaluation approach for the grasp posture based only on grasp stability does not necessarily ensure that the obtained grasp posture is truly "possible" one. There is a possibility that only using the grasp stability causes the wrong evaluation result. For example, the grasp posture in Table 1(b) satisfies the force-closure condition, and has high grasp quality value. Therefore, this posture has high grasp stability. But this grasp has the impossible grasp posture where the configuration of finger joint angles is quite different from the one we usually take in holding objects. Therefore, we need another index which can evaluate the validity of the configuration of finger joint angles of the grasp posture. We call this second index "ease of grasping (EOG)". Of course, only using this index

Table 1. The relation between grasp stability and ease of grasping

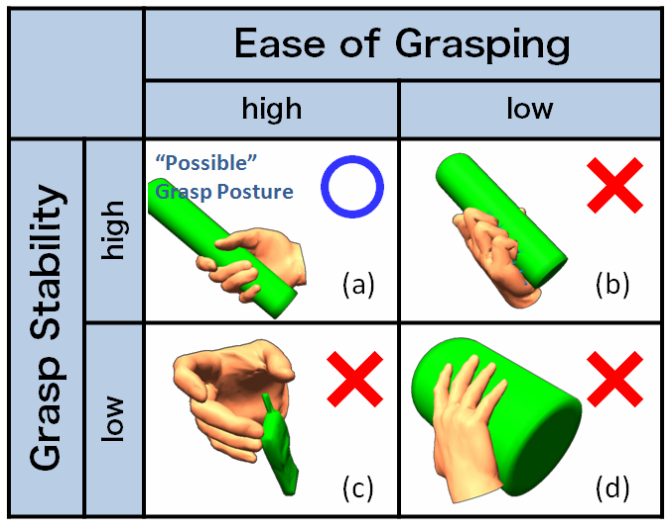


may sometimes cause the wrong evaluation results (Table 1(c)). Therefore, we define the truly "possible" grasp posture as the one which has high index value of the grasp stability and the ease of grasping, as shown in Table 1(a).

To evaluate the EOG, we have to calculate one index value at a certain configuration of finger joint angles. However, total degrees of freedom of fingers in a hand ranges from 25 to 32 [12], and it becomes very difficult to calculate an index value from the combination of these many finger angle values.

In neuroscience field, some research described that the finger joint angles of human hand are strongly interrelated with each other during the grasp motion and at holding state [13]. So the "possible" grasp postures are represented by less variables than the degree of freedom of the hand. Therefore, in this paper, we propose to apply this interrelation of the finger joint angles to decreasing the degrees of freedom of the hand and to constructing a new index for the grasp posture evaluation.

\section{The Proposed System for Generation and Evaluation of Grasp Posture}

In this section, we describe the method of how to evaluate the ease of grasping the object, which is the second evaluation index of the grasp. Figure 2 shows an overview of our evaluation method for the ease of grasping an object in our computer-aided ergonomic assessment system. The system consists of the following three steps:

1. As preprocess of the method shown in Figure 2(A1-A3), we construct an "ease of grasping evaluation map (EOG-map)" defined in an $M$-dimensional space, where a large number of actual hand postures from the opened state to the grasping state are plotted.

2. An initial estimated grasp posture is generated for a product model to be grasped (Figure 2(B1)), as described in our previous work [6].

3. The ease of grasping for the optimized grasp posture is evaluated (Figure 2(B2)).

We describe the above 1 and 3 in the following section.

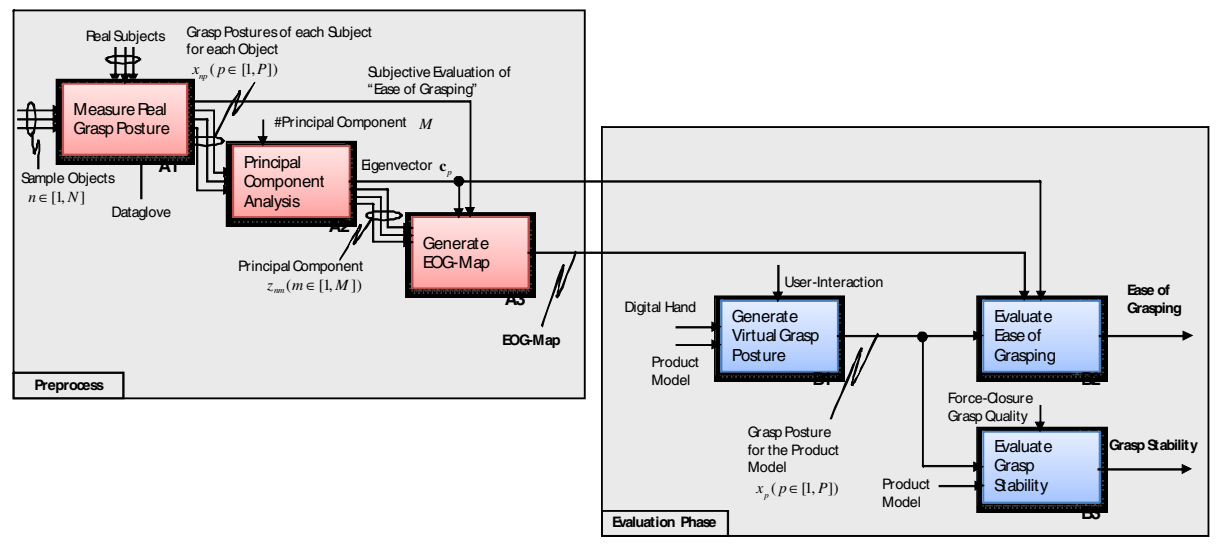

Fig. 2. The proposed system for evaluating ease of grasping 


\subsection{Building the EOG-Map}

The EOG-map consists of a set of plots in multi-dimensional space, and each of plot corresponds to a represent to finger joint angle configuration measured from real grasping examples. This plot is generated by the following process:

1. For a subject to grasp and an object to be grasped, a sequence of finger joint angles of the subject's hand from the opened state to the grasping state are measured.

2. A subject is asked to hold a set of objects including primitive shapes and real daily products.

3. A number of subjects are required to carry out the above experimental process of 1 and 2 .

4. All recorded sets of finger joint angles are processed by PCA and the results are plotted as points on the EOG-map.

In this map, one posture example is indicated as a set of scores of a principal component analysis (PCA) for finger joint angles of some "real" human hands, measured by a dataglove. We can estimate the ease of grasping which is generated from a product model and a digital hand by plotting the principal component score for this posture on the map.

\subsection{Principal Component Analysis for Finger Joint Angles of Hand Postures in Grasp Motion}

Let's define a set of $P$ joint angles of a hand posture as $\left\{x_{p} \mid p=1,2, \ldots, P\right\} . P$ indicates the degree of freedom of the hand model and is generally defined as more than 30. However, it has been known that finger joint angles are strongly interrelated with each other during the grasp motion [13]. Therefore, we reduce the degree of freedom of the hand posture to less than $P$ by PCA.

Suppose joint angles of the hand postures in grasping for $N$ objects are recorded. We define a set of the standardized measurements of these angles as $\left\{x_{n p} \mid n=1,2, \ldots, N ; p=1,2, \ldots, P\right\}$, and define a $N \times P$ matrix as $X=\left[x_{n p}\right]$ $\left(x_{n p} \in \mathfrak{R} ; n \in[1, N] ; p \in[1, P]\right)$. Then we obtain a variance-covariance matrix $V$ and the matrix $W=\left[w_{i j}\right]\left(w_{i j} \in \Re ; i, j \in\{1,2, \ldots, P\}\right)$ as

$$
\begin{gathered}
V=\frac{1}{N-1} X^{T} X, \\
W=\left[w_{i j}\right]=\left[\mathbf{c}_{1} \mathbf{c}_{2} \cdots \mathbf{c}_{P}\right],
\end{gathered}
$$

where $\mathbf{c}_{1}, \mathbf{c}_{2}, \ldots, \mathbf{c}_{P}$ are eigenvectors of the matrix $V$ which are sorted in decreasing order of eigenvalue.

Suppose the $P$ finger joint angles can be approximated by $M(M \leq P)$ principal components, then we approximately describe the hand posture as the first $M$ principal component scores $\mathbf{z}_{n}=\left[z_{n 1} z_{n 2} \ldots z_{n M}\right]$ as follows:

$$
z_{n m}=\sum_{p=1}^{P} w_{p m} x_{n p} \quad(m=1,2, \ldots, M, n=1,2, \ldots, N) .
$$




\subsection{Ease of Grasping Evaluation Map (EOG Map)}

\subsubsection{Method for Generating the EOG Map}

Using the above eq. 3, we can evaluate the sequence of the principal component scores $z_{n m}$ of the hand postures from the opened to grasping states for each sample object with "real" subjects. By plotting the first $M$ principal component scores of these postures in a $M$-dimensional space, we can generate the "ease of grasping evaluation map (EOG-map)". At the same time, we also record the two-level subjective evaluation for the grasp postures (i.e. "easy to grasp / not easy to grasp"). By partitioning the EOG-map into the equally spaced $M$-dimensional voxel, an EOG value eog_vox $(\gamma)$ is attached to each voxel $\gamma$. The range of this EOG value eog_vox $(\gamma)$ can be one of the following three values, which are:

1. easy_to_grasp - a voxel which includes grasp postures whose subjective evaluations are "easy to grasp"

2. able_to_grasp - a voxel which includes hand postures during the grasping sequence but does not include final grasp postures

3. unable_to_grasp - a voxel which includes neither hand postures nor grasp postures

Therefore, the function eog_vox $(\gamma)$ is defined as

$$
\text { eog_vox }(g): \Gamma \rightarrow\{\text { easy_to_grasp, able_to_grasp, unable_to_grasp }\}
$$

where, $\Gamma$ is a set of all voxel.

\subsubsection{Generation of the Multiple EOG-Maps Classified by the Hand Dimension}

There is a possibility that a large variance in the hand sizes of the subjects causes different results in the EOG evaluation. To consider this difference, we classify subjects into five groups with respect to their hand dimension and generate five different EOG-maps for the five groups. In the measurement of the preprocess, we prepare nine sheets of the paper where the nine representative Japanese hands shown in Figure 3(a) are printed. By putting the subject's hand on these sheets, each subject chooses one representative hand that has the most equivalent hand dimension. Based on the class of their chosen representative hand, the dimensions of subject's hand are classified into five groups, as shown in Figure 3(b).

\subsubsection{Results of Generating the EOG Map}

Figure 4(a) shows one of the EOG evaluation maps generated by the above method (the $5^{\text {th }}$ hand group in Figure 3(b)). The blue/red circular points show the grasp postures that have the subjective evaluation of "easy to grasp / not easy to grasp". The small gray points show the instantaneous hand postures in grasping sequence. The EOG evaluation maps are generated by 8 subjects and 70 sample grasped objects, as shown in Figure 4(b). The finger joint angles of this hand postures were measured by the "CyberGlove", which has 19 sensors. We determined that the number $M$ of the 


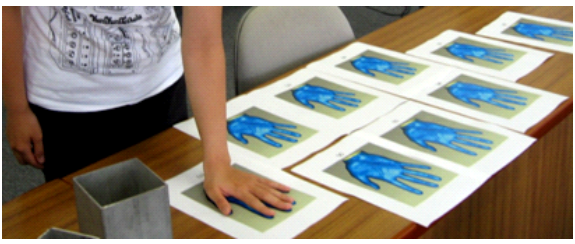

(a)

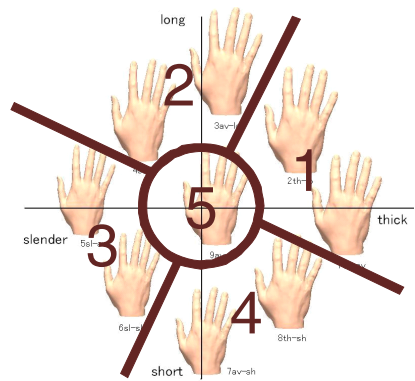

(b)

Fig. 3. The generation of multiple EOG-map classified by the hand dimension

principal components should be three, because the cumulative proportion of the first three principal components was more than $80 \%$ at every EOG evaluation map of each hand group.

\subsection{The Evaluation and Verification for Ease of Grasping}

In the evaluation process in Figure 2(B2), the optimized grasp posture $i$ of the digital hand is generated, and the vector of the principal component scores $\mathbf{z}_{i}$ for this posture $i$ is calculated from eq. 3. Then, the ease of grasping for this grasp posture $i \operatorname{eog}(i)$ can be defined as $\operatorname{eog}(i)=\operatorname{eog} \_\operatorname{vox}\left(\gamma^{i}\right)$ where $\gamma^{i}$ is a voxel including a point $\mathbf{z}_{i}$, and the function eog_vox $(\gamma)$ returns one of the EOG values of the voxel $\gamma$ as defined in the previous section.

We verified the ease of grasping evaluation by plotting some grasp postures of the digital hand on the EOG-map, as shown in Figure 5. These grasp postures are generated from the digital hand and the product models which have the same geometry as the real shape of the product used in generating the EOG-map. The blue/red rectangle points show the principal component scores of the grasp postures
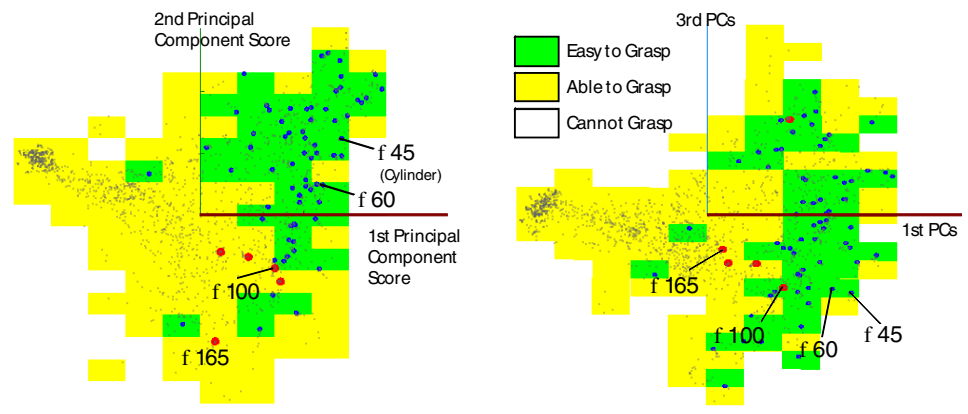

(a)

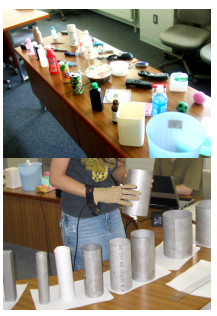

(b)

Fig. 4. (a) The EOG map and (b) sample objects used for generating the EOG-map 

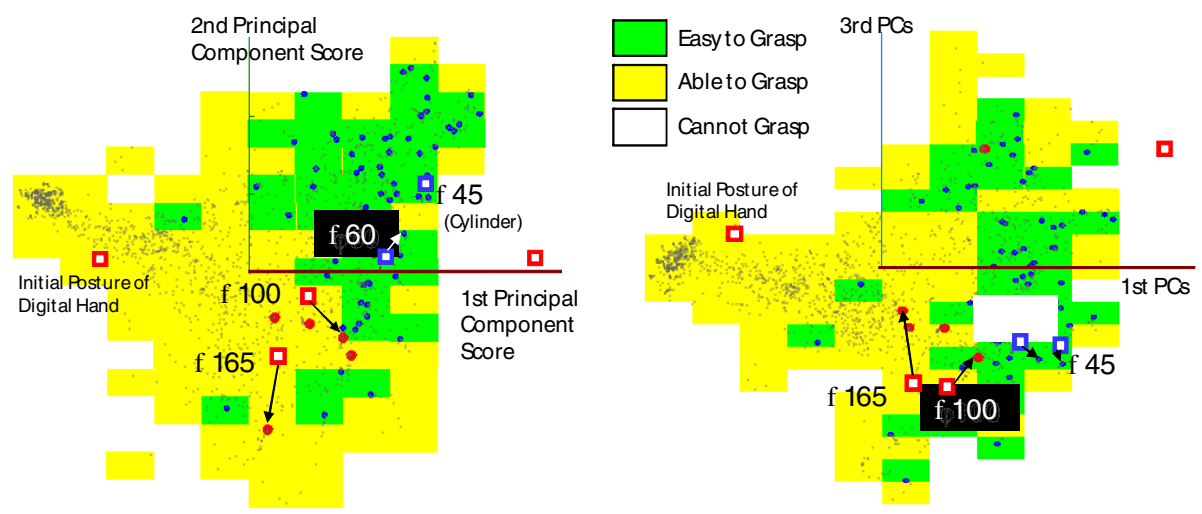

Fig. 5. The evaluation and verification results of the ease of grasping

that were estimated as "stable/unstable" grasp posture by force-closure. The two-point pair connected with an arrow shows that these grasp postures are for the same grasped object. The EOG evaluation results by taking a digital hand and by a real subject's hand for the same object fell into the same category for almost all cases. Therefore, the proposed EOG-map-based method is effective for evaluating the ease of grasping a product model with a digital hand.

\section{Conclusions}

The conclusions of our research summarizes as follows:

1. We proposed a system of automatic ergonomic assessment for handheld information appliances by integrating the digital hand model with the 3dimensional product model of the appliances.

2. The ease of grasping evaluation (EOG) map was introduced by measuring the grasp postures from real subjects. Based on this EOG map, we proposed an evaluation method of ease of grasping from the product model and the digital hand model. The EOG values by taking a digital hand and by a real subject's hand for the same object fell into the same classification of the easiness. PCA for the finger joint angles enables to calculate the posture similarity with much less variables than the DOF of the human hand.

In our future research, we will develop a new function to evaluate the easiness of finger operation for the use-interface of the products, and to aid the designers in redesigning the housing shapes and the user-interfaces in the product model.

Acknowledgements. This work was financially supported by a grant-in-aid of Intelligent Cluster Project (Sapporo IT Carrozzeria) funded by MEXT. 


\section{References}

1. For example, Jack.: http://www.ugs.com

2. Kouchi, M. et al.: An Analysis of Hand Measurements for Obtaining Representative Japanese Hand Models. In: Proceedings of the 8th Annual Digital Human Modeling for Design and Engineering Symposium, 2005-01-2734 (2005)

3. Carozzeria, S.T.: http://www.it-cluster.jp/english/

4. Miller, et al.: GraspIt!: A Versatile Simulator for Robotic Grasping. IEEE Robotics and Automation Magazine, 110-122 (2004)

5. Kyota, F., et al.: Detection and Evaluation of Grasping Positions. In: Proceedings of the 2005 ACM SIGGRAPH, sketches (2005)

6. Endo, Y., et al.: An Application of a Digital Hand to Ergonomic Assessment of Handheld Information Appliances. In: Proceedings of SAE 2006 Digital Human Modeling for Design and Engineering Conference, 2006-01-2325 (2006)

7. Yang, J., et al.: Posture Prediction and Force/Torque Analysis for Human Hands. In: Proceedings of the SAE 2006 Digital Human Modeling for Design and Engineering Conference, 2006-01-2326 (2006)

8. Miyata, N., et al.: Modeling of Human Hand Link Structure from Optical Motion Capture Data. IEEE/RSJ Int. Conf. Intelligent Robots and Systems, pp. 2129-2135 (2004)

9. Miyata, N., et al.: Method to Derive Hand Link Model with MRI images. In: Proceedings of the 21st Annual Conference of the Robotics Society of Japan, 1A11 (2003)

10. Nguyen, V.: Constructing force-closure grasps. The International Journal of Robotics Research 7(3), 3-16 (1988)

11. Pollard, N.S.: Closure and Quality Equivalence for Efficient Synthesis of Grasps from Examples. The International Journal of Robotics Research 23(6), 595-613 (2004)

12. Lee, J., et al.: Model-Based Analysis of Hand Posture. IEEE Computer Graphics and Applications 15(5), 77-86 (1995)

13. Santello, M., et al.: Postural Hand Synergies for Tool Use. The Journal of Neuroscience 18(23), 10105-10115 (1998) 